\title{
ESTUDIO ULTRASONOGRÁFICO DE LAS MASAS RENALES DE PEQUEÑO TAMAÑO.
}

\author{
Enrique Sanz Mayayo, Ricardo García Navas, Rafael Rodríguez-Patrón Rodríguez, \\ Fernando Arias Fúnez, Alberto Lennie Zuccarino y Teodoro Mayayo Dehesa.
}

Unidad de Ecografía Urológica. Hospital Ramón y Cajal. Madrid. España.

\begin{abstract}
Resumen.- OBJETIVO: Efectuar una puesta al día de la utilidad de la ecografía en el estudio de los tumores renales de pequeño tamaño y de las posibilidades que esta técnica ofrece en el momento actual.

MÉTODO: Revisión de los resultados obtenidos en esta patología con las aportaciones ofrecidas por los desarrollos tecnológicos más recientes como los convertidores digitales con modo armónico y el Doppler color energía. Análisis de su contribución al diagnóstico diferencial con la patología quística, a la definición de las masas sólidas, a la detección y caracterización de las masas de pequeño tamaño y a la definición de los patrones vasculares de los diferentes tumores.
\end{abstract}

RESULTADOS: La ecografía ofrece una seguridad diagnóstica del $98 \%$ en las masas quísticas pudiéndose ob- jetivar en condiciones favorables a partir de un diámetro de 0,5 cm. El diagnóstico diferencial de las masas multitabicadas, quiste hidatídico multivesicular, nefroma quístico multilocular y carcinoma quístico multilocular, sigue planteando graves dificultades, al igual que ocurre con el resto de las técnicas de imagen. En las masas sólidas la mayor resolución de las imágenes ha conducido a un incremento progresivo en la detección de tumores incidentales y en el porcentaje de pacientes candidatos a cirugía conservadora dada la continua disminución en su tamaño. La diferenciación entre el adenocarcinoma y el angiomiolipoma es fácil y posible en el $85 \%$ de los casos no presentando el resto de tumores características específicas. En las masas de pequeño tamaño, menores de $3 \mathrm{~cm}$., la sensibilidad de esta técnica es claramente inferior a la de la TAC. El Doppler color energía ayuda a confirmar la existencia de las masas sólidas y permite un mejor diagnóstico diferencial con los seudotumores.

CONCLUSIONES: Las modernas técnicas ecográficas ofrecen una elevada rentabilidad tanto en la detección como en la definición de la naturaleza de las masas renales de pequeño tamaño.

Palabras clave: Ecografía. Masas renales. Ecografía urológica.

Summary.- OBJECTIVES: To perform an update on the usefulness of ultrasound in the study of small size renal tumors and its current possibilities.

METHODS: We review the results of ultrasound in this pathology with the addition of the most recent technological advances such as a harmonic digital converters and power color Doppler. We analyze its contribution to the differential diagnosis of the cystic pathology, to the 
definition of solid masses, to the detection and characterization of small size masses, and to the definition of the vascular patterns of various tumors.

RESULTS: Ultrasonography offers a diagnostic safety of $98 \%$ in cystic masses, being able to detect them from $0.5 \mathrm{~cm}$ diameter in favourable conditions. The differential diagnosis of multiloculated masses, multivesicular hydatid cyst, multiloculated cystic nephroma, and multiloculated cystic carcinoma still poses great difficulty, the same way it happens with other radiological tests. For solid masses, the greater image resolution has lead to a progressive increase in the incidental detection of tumors and the percentage of patients candidates to conservative surgery due to the decrease in size. It is easy to differentiate between adenocarcinoma and angiomyolipoma, up to $85 \%$ of the cases, but the rest of the tumors do not have specific characteristics. For small size masses, smaller than $3 \mathrm{~cm}$, ultrasound sensitivity is clearly inferior to CT scan. Power color Doppler helps to confirm the existence of solid masses and helps a better differential diagnosis with pseudo tumors.

CONCLUSIONS: The modern ultrasound techniques provide a high cost-effectiveness both in detection and definition of the nature of small size renal masses.

Keywords: Ultrasonography. Renal masses. Urological ultrasound.

\section{INTRODUCCIÓN}

Durante los últimos veinte años la incidencia del carcinoma de células renales (CCR) se ha incrementado debido a la extensión del uso de técnicas de exploración abdominal no invasivas como la ecografía, TAC y resonancia nuclear magnética. De forma paralela ha aumentado el número de hallazgos incidentales. Esto va asociado a una disminución del tamaño tumoral en el momento de la detección y a un mejor pronóstico. A pesar del escaso progreso experimentado en el tratamiento la supervivencia a 5 años en pacientes con esta patología ha mejorado significativamente desde el $37 \%$ en los diagnosticados a principios de los sesenta al $52 \%$ entre 1974 y 1976 y el $58 \%$ entre 1983 y 1989 (1). Esto es debido en gran parte a la notable mejoría en el diagnóstico radiológico que permite la detección en estadios más tempranos de la enfermedad.

El desarrollo de estas nuevas técnicas ha permitido definir una nueva entidad clínica que es el tumor incidental o accidentalmente descubierto en el curso de una exploración rutinaria con otra finali- dad habiéndose reportado que por encima del $80 \%$ de ellos son detectados mediante ecografía (2). La tasa de los así diagnosticados ha aumentado sistemáticamente en los últimos 30 años. Para Skinner (3) fue del 7\% en 1971 y Konnak (4) en 1985 comparando los datos antes y depués de la llegada de la ecografía comprobó un incremento del 13 al $48 \%$. Bretheau (5) encontró una progresión similar desde el $17 \%$ de 1980 al $48 \%$ de 1991 . En la serie de Patard (6) aumento del $10 \%$ en 1984 hasta el $52 \%$ en 1999. Esto no es sorprendente ya que en un estudio que analiza las autopsias realizadas entere 1958 y 1969 el $70 \%$ de los adenocarcinomas renales se descubrieron en pacientes en los que no se sospechó clínicamente (7). Paralelamente se ha comprobado una significativa reducción del tamaño tumoral y un considerable aumento en la proporción de pacientes candidatos a cirugía conservadora, con una mejoría en la supervivencia, a 5 años de 85 al $90 \%$, frente al $30-60 \%$ entre los sintomáticos $(8,9)$. Por lo tanto el descubrimiento incidental del carcinoma renal debe considerarse como un factor pronóstico añadido al grado y estadio.

Se definen como masas renales de pequeño tamaño las menores de 3 centímetros de diámetro mayor. Un porcentaje elevado de éstas corresponden a lesiones quísticas, que requieren una evaluación muy cuidadosa ya que a veces son difíciles de diferenciar de los CCR en estadio iniciales. En general estas masas suponen un reto diagnóstico en su detección y caracterización para el conjunto de las técnicas de imagen.

El estándar-oro para el estudio de los CCR es la TAC, pero la ecografía ejerce un papel muy importante en la detección precoz; El 83\% de los tumores renales asintomáticos son descubiertos incidentalmente mediante ecografía (2). Las limitaciones de esta van ligadas por un lado al tamaño, localización y ecogenicidad de la lesión y por otro a las características físicas del paciente como la obesidad y la presencia de gas intestinal. Así, tumores de pequeño tamaño isoecoicos intraparenquimatosos necesitan para poder ser detectados que el crecimiento modifique el contorno renal. También puede ser difícil la visualización de tumores de polo inferior con crecimiento exorrenal en paciente con abundante gas intestinal.

Con el modo armónico se obtiene una mejor definición de los bordes de las estructuras y órganos con una mejor demostración del contenido y de la resolución del contraste con menos artefactos en la escala de grises. Con el Doppler color, asociado al Doppler energía, conocemos la vascularización mejorando la sensibilidad en la detección de las peque- 
ñas masas renales. La utilización de contrastes ultrasónicos, que actúan como potenciadores de señal, produce un aumento de la respuesta vascular de la cortical y puede poner de manifiesto mínimas lesiones ocupantes (10). Otras posibilidades como la cuantificación de la respuesta ecogénica de las imágenes digitalizadas midiendo el valor de los grises puede también ayudar a la diferenciación entre pequeña masa de dudosa naturaleza, como los carcinomas hiperecogénicos y los angiomiolipomas (11).

\section{DIAGNÓSTICO DIFERENCIAL: MASAS QUÍSTICAS.}

El contenido habitualmente uniformemente líquido de las diferentes variedades de quistes renales permite obtener imágenes patognomónicas fácilmente reconocibles (Figuras 1 y 2 ).

Los corticales aislados o múltiples tienen unas paredes perfectamente dibujadas con un refuerzo de las estructuras posteriores y un contenido anecoico con ocasionales finos tabiques aislados (Figura 3). Los intrasinusales alcanzan menor volumen y son múltiples y con frecuencia bilaterales en las hiliosinuquistosis. Las poliquistosis producen patrones característicos con riñones aumentados de tamaño, desestructuración global de los parénquimas y senos en los casos morfológicamente avanzados y efecto de "panel de abejas".

En la actualidad la ecografía permite una seguridad diagnóstica de prácticamente el $98 \%$ en las masas quísticas no siendo precisa ninguna otra exploración en ausencia de sintomatología (12). Pueden detectarse, en condiciones anatómicas favorables a partir de un diámetro superior a $0,5-1 \mathrm{~cm}$.

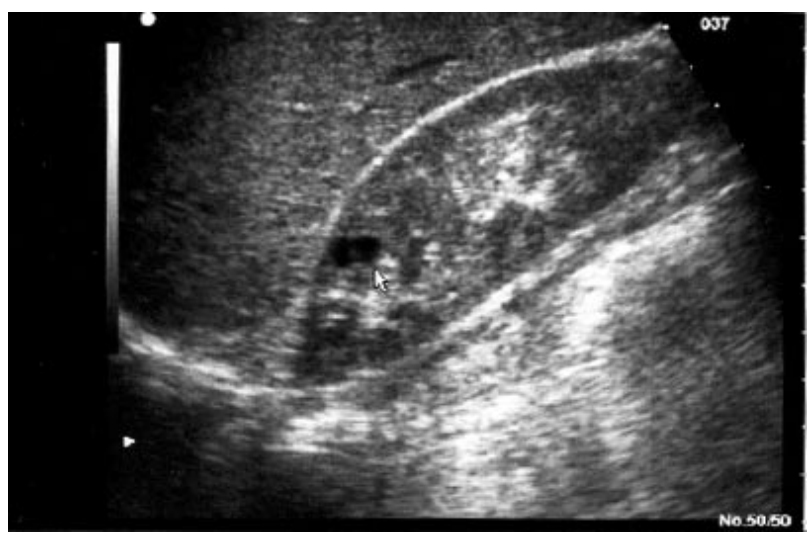

FIGURA 1. Sección longitudinal: dos quistes corticales contiguos a nivel del polo superior de aproximadamente $0,5 \mathrm{~cm}$ de diámetro (flecha).
Especial problema plantean algunas entidades como los infrecuentes quiste hidatídicos o el nefroma quístico multilocular también denominado quiste multilocular congénito.

La hidatidosis renal es rara aun en los países endémicos; supone del 1,9 al 7,2\% de todas las localizaciones del quiste hidatídico (13) siendo las más frecuentes la hepática y la pulmonar. La ecografía ofrece imágenes demostrativas que orientan hacia el diagnóstico en la mayoría de los casos. Han sido descritos varios patrones superponibles a los obtenidos en otros órganos (14-18).

El tipo I, colección líquida pura, corresponde al univesicular, el menos frecuente en la localización renal, puede ser indistinguible del seroso ayudando a su sospecha la presencia de engrosamiento parietal o calcificación. En una serie de Odev (19) de 17 pacientes se objetivó en el $21 \%$. El tipo II es generalmente específico presentando una pared desdoblada, dislacerada con imagen de membrana flotante producida por la separación de la germinativa. EI tipo III también es característico y corresponde al multivesicular con tabiques internos mas o menos gruesos y ecogénicos y múltiples lóculos producidos por las vesículas hijas reproduciendo una imagen en "panal de abejas". Es la forma más frecuente de presentación. El tipo IV tiene una morfología seudotumoral debido a la presencia de membranas rotas, escólices y granos hidatídicos que pueden producir un aspecto aparentemente sólido muy difícil de diferenciar de un adenocarcinoma. El tipo $\boldsymbol{V}$, pared quística hiperecoíca producida por las calcificaciones, es sugestivo de áreas endémicas.

Si bien en el hígado el Ill se considera patognomónico en el riñón pueden presentar esta misma

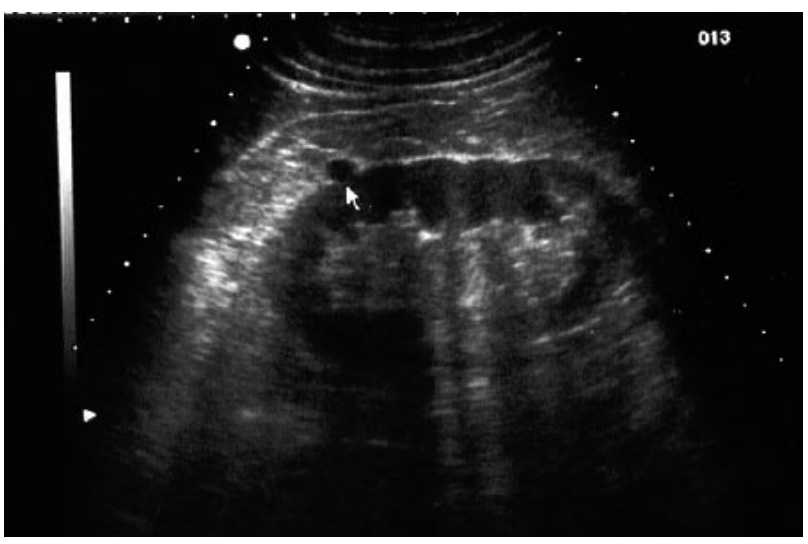

FIGURA 2. Sección longitudinal: quiste seroso en polo superior de 0,5 cm (límite de resolución). 


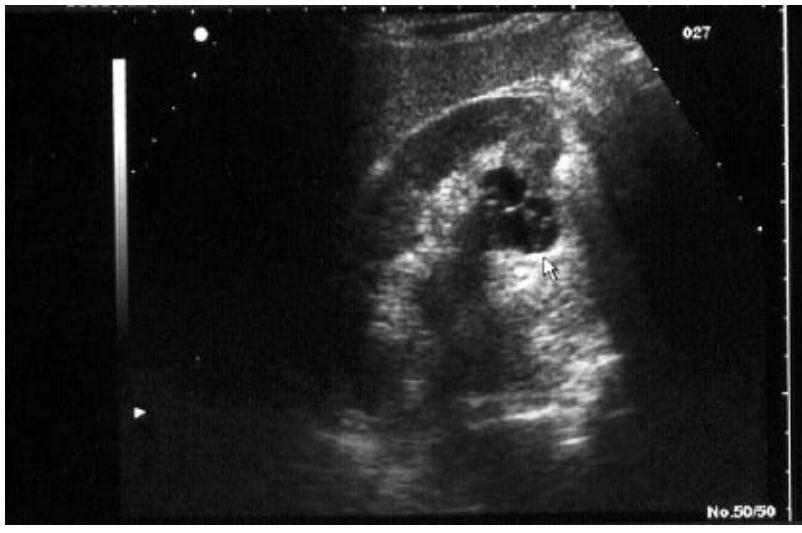

FIGURA 3. Sección longitudinal: quiste seroso tabicado de aproximadamente $3 \mathrm{~cm}$ (flecha).

morfología otras masas multitabicadas como el quiste seroso paucilocular, el carcinoma quístico multilocular o el nefroma quístico multilocular. El último considerado actualmente como un tumor de comportamiento benigno habitualmente no produce sintomatología y es unilateral, único, con quistes no comunicados entre si ni con la vía excretora.

\section{DIAGNÓSTICO DIFERENCIAL: MASAS SÓLIDAS Y MIXTAS.}

En nuestra experiencia la mayor proporción de masas sólidas descubiertas incidentalmente con ecografía han correspondido a angiomiolipomas, generalmente pequeños, aislados y en mujeres. El resto han presentado una distribución histológica si-

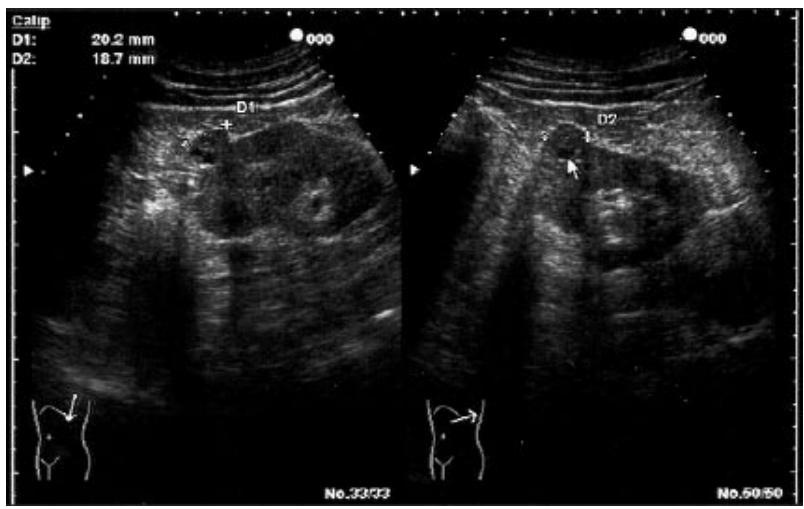

FIGURA 5. A: Sección longitudinal. B: Sección transversal. Masa sólida de $2 \mathrm{~cm}$ con alguna área líquida en su interior (flecha).

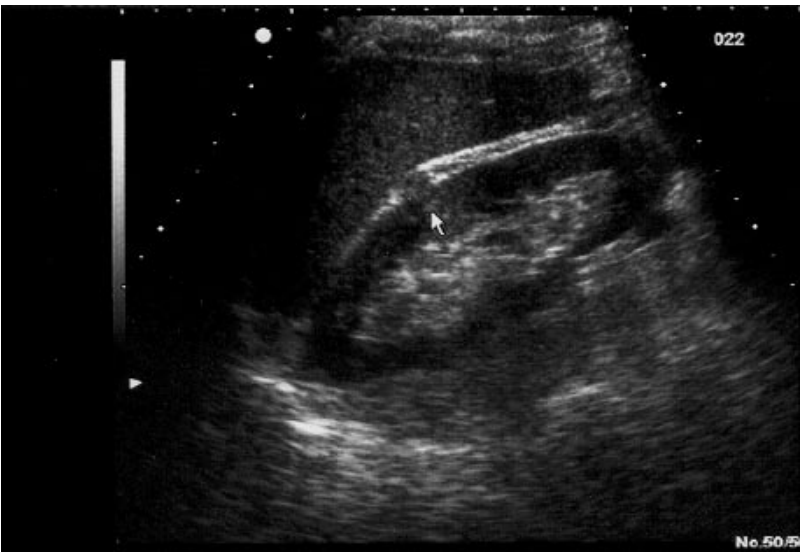

FIGURA 4. Sección longitudinal: masa sólida a nivel de tercio medio de $1.5 \mathrm{~cm}$ que modifica el contorno renal (flecha).

milar a las sintomáticas correspondiendo del 90 al 95\% a CCR con un mínimo porcentaje de oncocitomas, leiomiomas, fibromas, linfomas $u$ otros tumores mesenquimales benignos o malignos.

El adenocarcinoma renal no tiene desde el punto de vista ultrasonográfico unas características homogéneas (Figura 4). Generalmente da lugar a ecos de diferentes intensidades con una distribución heterogénea e irregular con amputación del parénquima y ecos sinusales a nivel de la zona de desarroIlo (Figuras 5-8). En sus formas más típicas su ecogenicidad es ligeramente mayor que la del parenquima renal y en su interior pueden existir pequeñas áreas transónicas producidas por focos necróticos o hemorrágicos (Figura 9). Aunque podría esperarse que los muy vascularizados fueran hiperecoicos y los avascu-

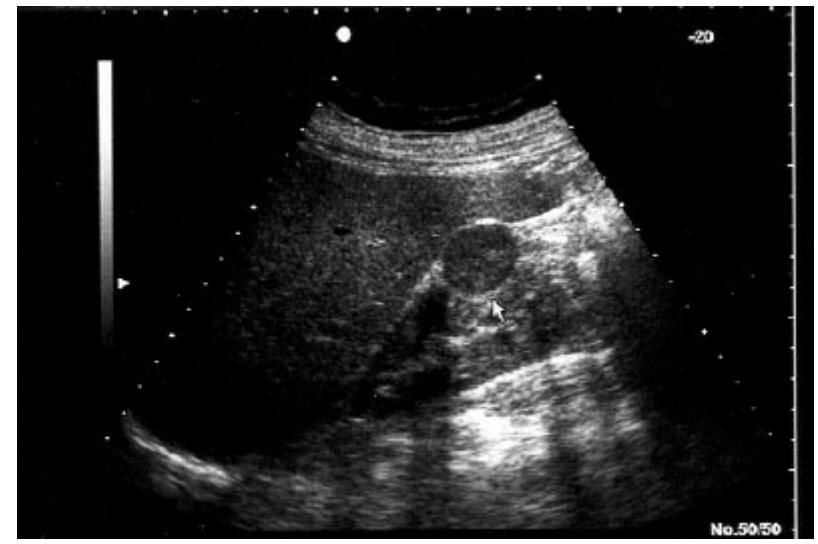

FIGURA 6. Sección longitudinal: Masa sólida de $3 \mathrm{~cm}$ de ecogenicidad algo mayor que el parenquima renal (flecha). 


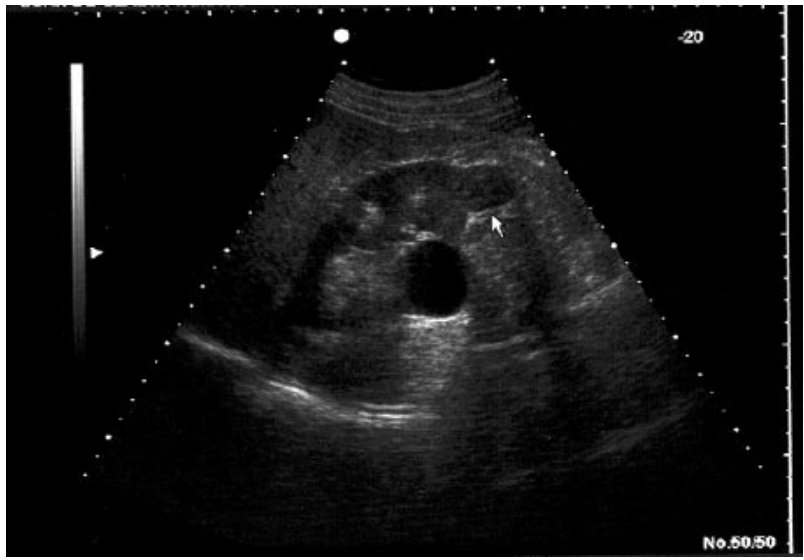

FIGURA 7. Sección longitudinal: Coexistencia de quiste seroso en tercio medio y masa sólida en polo inferior de aproximadamente $2 \mathrm{~cm}$ (flecha).

lares más anecoicos no hemos encontrado clara relación entre la respuesta acústica y la vascularización.

Los tumores calcificados pueden presentar dificultades diagnósticas a veces insalvables. Cuando las calcificaciones son periféricas y lineales es posible objetivar la estructura interna pero cuando son gruesas e irregularmente distribuidas puede ser imposible definirla (Figuras 10 y 11 ).

El diagnóstico diferencial entre el CCR y el resto de las masas sólidas renales de contenido no graso es imposible. Se ha descrito en el oncocitoma un patrón característico (20) parecido al obtenido

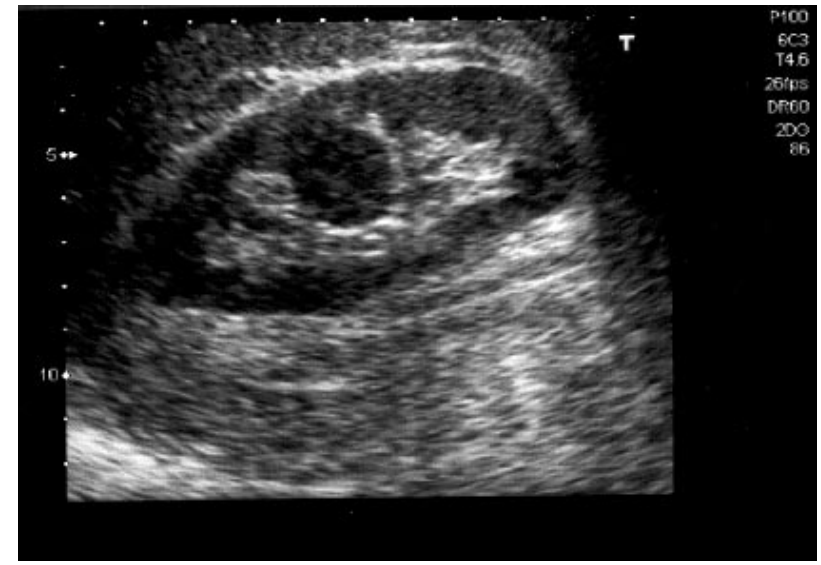

FIGURA 8. Sección longitudinal: Masa sólida de $3 \mathrm{~cm}$ que penetra en el seno, diagnostico diferencial con tumor transicional.

con la TAC. La delimitación de un área hipoecoica central estrellada traduce la presencia de la cicatriz característica de este tumor que globalmente presenta una respuesta uniforme con bordes bien definidos, lisos y lineales. Aunque sugestivos, estos signos no son patognomónicos y no permiten un diagnóstico de seguridad habiendo presentado, en nuestra experiencia, algunos carcinomas parecido aspecto.

Aproximadamente el $85 \%$ de los angiomiolipomas aparecen como masas redondeadas, intensamente ecogénicas, de aspecto algodonoso, fácilmente diferenciables del parénquima normal (Figuras 12 y 13). Cuando su tamaño es significativo sus bordes

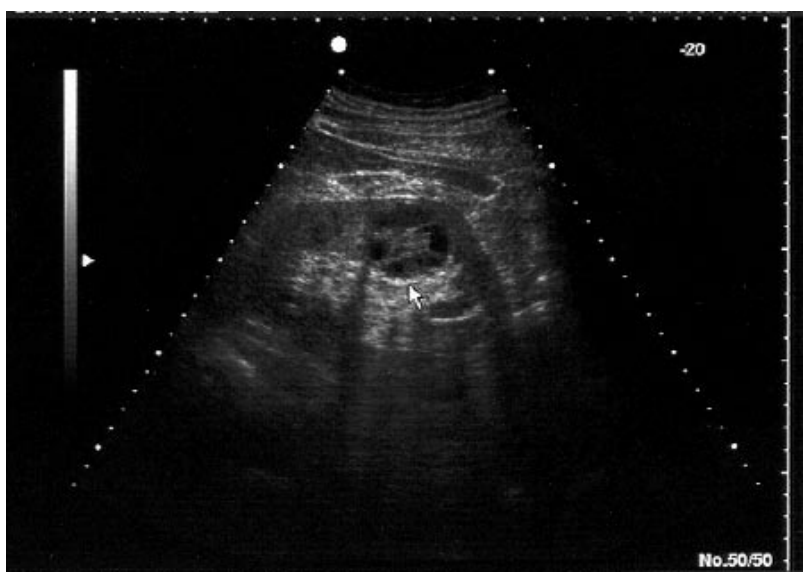

FIGURA 9. Sección longitudinal: Masa intraparenquimatosa heterogénea con áreas líquidas y sólidas en su interior (flecha).

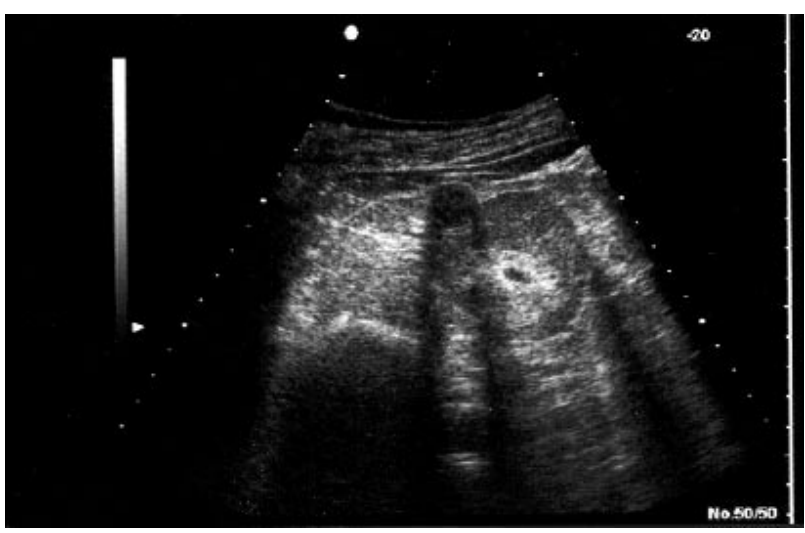

FIGURA 10. Sección longitudinal: Masa sólida en tercio medio con calcificación parietal que deja sombra acústica (flecha). 


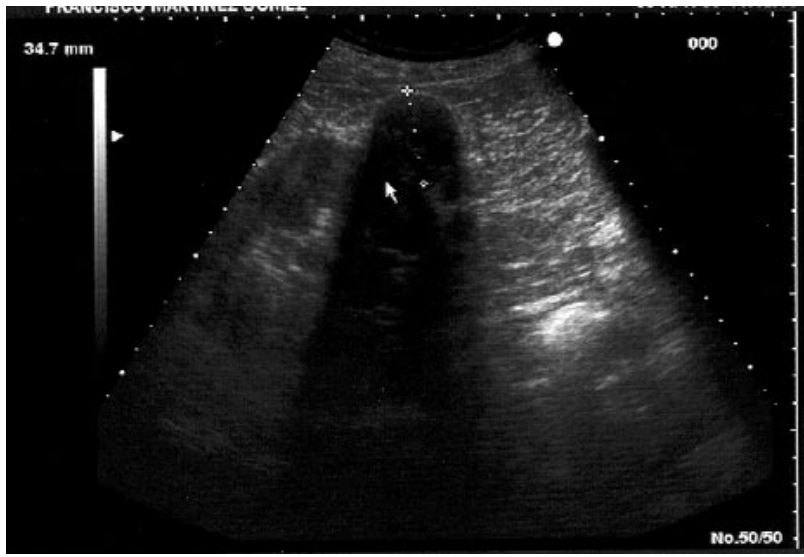

FIGURA 1 1. Sección longitudinal: Masa sólida calcificada con sombra acustica que impide su correcta visualización (flecha).

pueden ser difíciles de definir al integrarse en la misma ecogenicidad que la grasa perirrenal. El resto, posiblemente debido al elevado contenido de músculo o a la presencia de áreas hemorrágicas produce imágenes más heterogéneas y menos características. La existencia de algunos carcinomas altamente ecogénicos, obliga, por otra parte, a confirmar el diagnóstico mediante TAC. En su seguimiento posterior la ecografía será suficiente para monitorizar posibles variaciones en su tamaño y morfología.

Mayores dificultades interpretativas presentan las llamadas masas mixtas al no cumplir estrictamente los criterios de las quísticas ni de las sólidas. Plantean un singular desafío diagnóstico tanto para la ecografía como para el resto de las técnicas

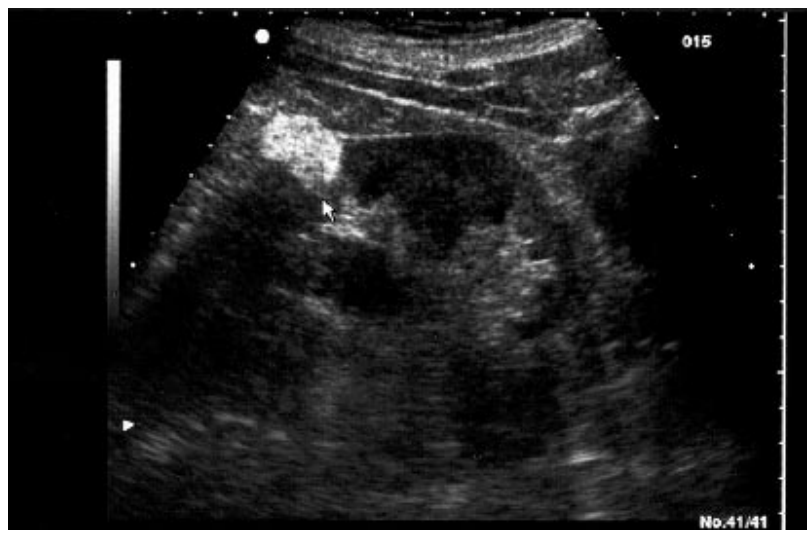

FIGURA 13. Sección longitudinal: Angiomiolipoma de $3 \mathrm{~cm}$ a nivel de polo superior (flecha).

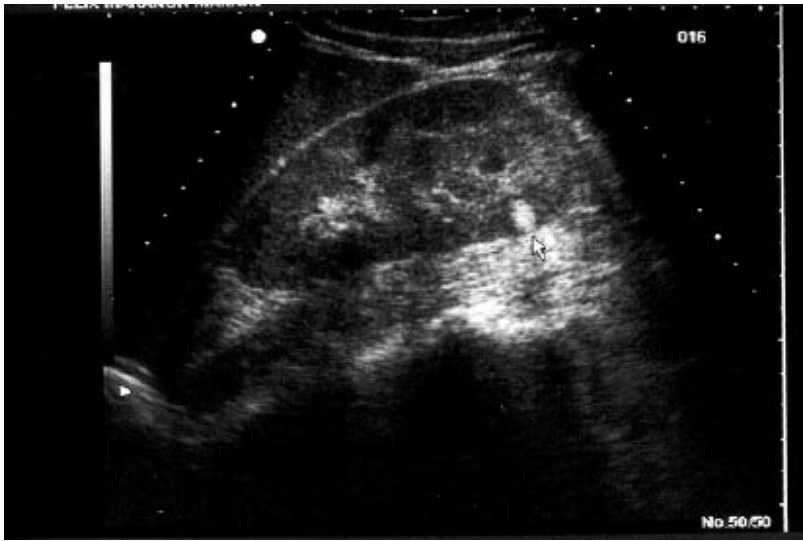

FIGURA 12. Sección longitudinal: Pequeño angiomiolipoma a nivel de polo inferior de aproximadamente $1,5 \mathrm{~cm}$ (flecha)

de imagen. Pueden ser uniloculares (sin tabiques) o multiloculares (con múltiples tabiques). Las primeras pueden presentar paredes gruesas, calcificaciones y ecos internos dispersos y pueden corresponder a adenocarcinomas necróticos, cistoadenocarcinomas, adenocarcinomas de tipo papilar, hemorragias 0 infecciones intraquísticas, abscesos o a los extraordinariamente infrecuentes carcinomas en las paredes de quistes serosos. Ninguno de los procedimientos diagnósticos permiten una segura diferenciación no excluyendo la malignidad la citología aspirativa negativa.

Entre las multiloculares cuando el contenido es claramente líquido y seroso y las paredes y tabiques bien definidos y claros con alta probabilidad

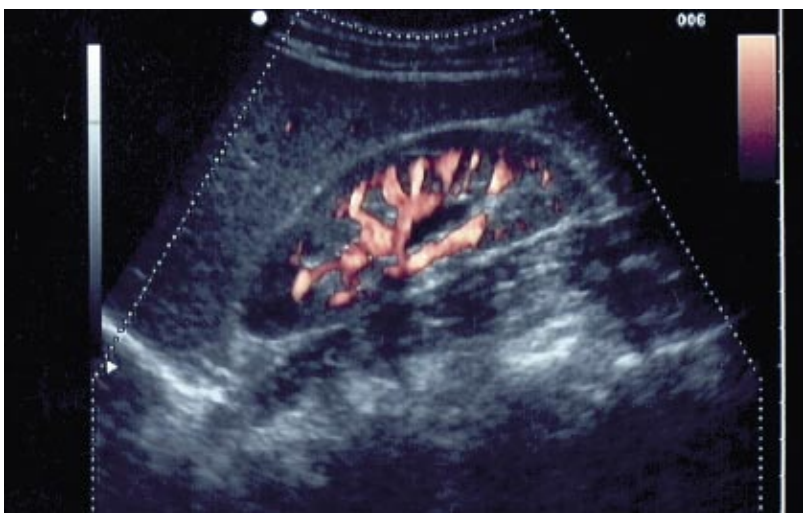

FIGURA 14. Sección longitudinal: Vascularización renal normal con Doppler. 


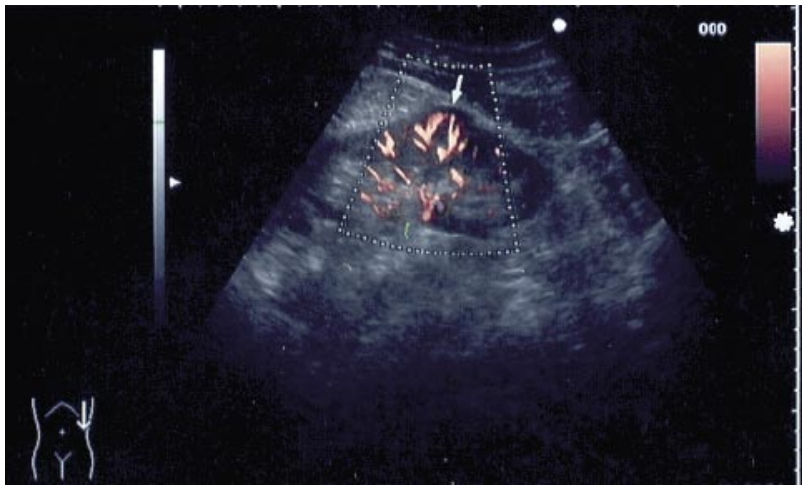

FIGURA 15. Sección longitudinal: Lobulación fetal, no se modifica la vascularización renal normal con Doppler (flecha).

estaremos en presencia de un quiste seroso tabicado que únicamente precisará evolución periódica. Cuando las paredes sean gruesas, los lóculos múltiples con zonas ecogénicas internas, con o sin calcificaciones, puede tratarse de un quiste complicado, de un quiste hidatídico multivesicular, de un nefroma quístico multilocular o de un adenocarcinoma quístico multilocular permitiendo únicamente la cirugía su correcta identificación.

Bosniak (21) clasifica estas masas, según su aspecto en la TAC, en cuatro categorías diferenciando en cada una de ellas una serie de signos en gran parte extrapolables a los obtenidos con la ecografía; en las dos primeras incluye los quistes típicos, mínimamente complicado y algunos hemorrágicos en

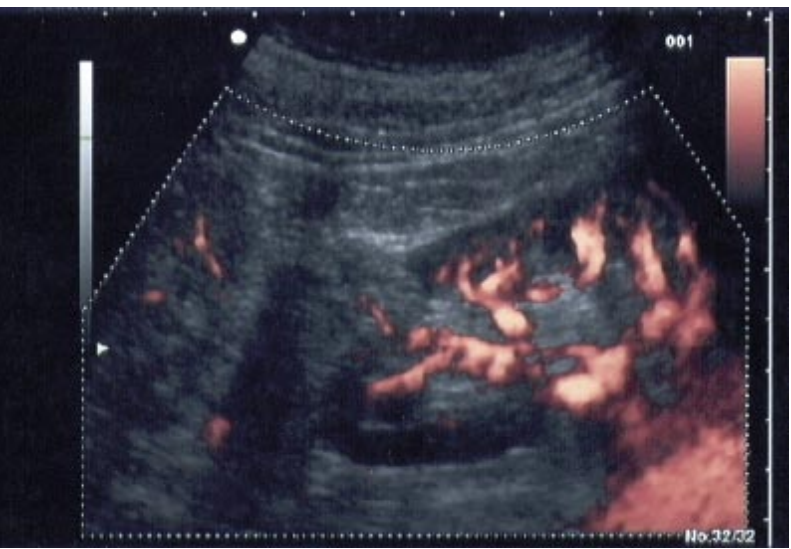

FIGURA 17. Sección longitudinal: Masa renal con vascularización central en Doppler.

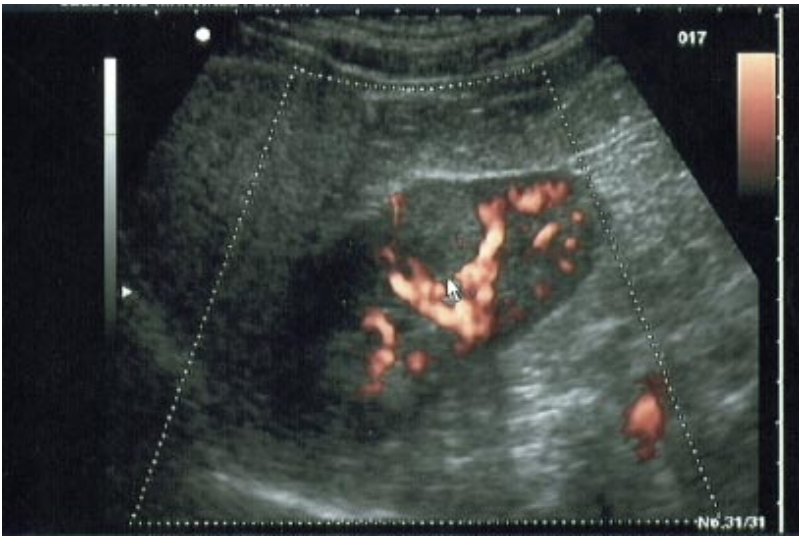

FIGURA 16. Sección longitudinal: Masa renal en tercio medio con vascularización periférica y ausencia de vascularización central (flecha).

los que en base a las imágenes puede evitarse la intervención quirúrgica, siendo ésta necesaria en las dos últimas, en las que se integran los carcinomas quísticos uni o multiloculares y los quistes más complicados.

En pacientes candidatos a cirugía conservadora por riñón único o limitado volumen tumoral es de gran interés, además de la determinación precisa del tamaño, localización y forma de crecimiento, establecer su posible multifocalidad. A este respecto la ecografía tiene una sensibilidad limitada, el $40 \%$ en la serie de Schlicker (22), debido a la dificultad de detectar los tumores adicionales menores de $2 \mathrm{~cm}$ y la imposibilidad por debajo de $1 \mathrm{~cm}$. Con la TAC helicoidal con cortes finos y reconstrucción multiplanar los resultados son claramente superiores.

\section{COMENTARIOS}

Globalmente la ecografía presenta una sensibilidad aproximada del $70 \%$ en la detección de tumores renales de pequeño tamaño con un límite de resolución de 1,5 centímetros y la TAC del 55\% con un límite de 1 centímetro. Jamis-Dow (23) en su estudio de 20 pacientes con enfermedad de Von-HippleLindau y con carcinoma renal hereditario comparó la eficacia de la TAC y la ecografía para la detección de este tipo de masas. La TAC detecto el $47 \%$ de los menores de $5 \mathrm{~mm}$ y prácticamente todos de $1,5 \mathrm{a}$ $2 \mathrm{~cm}$; sin embargo la ecografía no detectó ninguna menor de $5 \mathrm{~mm}$ y el $58 \%$ de las de 1,5 a $2 \mathrm{~cm}$.

El Doppler energía (power Doppler) puede ser de ayuda para la detección de estas pequeñas masas renales ya que permite una mejor representa- 
ción de la señal sobre todo con el uso de potenciadotes intravenosos con microburbujas que producen un efecto policromático; su utilidad práctica todavía está por definir (Figuras 14-17). Jinzaki (24) evaluó las características ultrasónicas y distribución vascular de 64 masas sólidas de entre 1,5 y $3 \mathrm{~cm}$, intentando correlacionar el resultado histopatológico con los hallazgos del Doppler energía. Describió cuatro modelos diferentes según la distribución vascular:

$0:$ Ausencia de señal.

1: Señales intratumorales focales.

2: Vasos penetrantes.

3: Distribución vascular periférica.

4: Mixto, penetrante y periférica.

De los 26 CCR, 24 eran del modelo 4 y 2 del 3 , en ninguno de ellos se vio distribución 0,1 o 2. De los angiomiolipomas, 24 tuvieron el 1, 3 el 2,2 el 3 y 5 el 4 . Los oncocitomas no presentaban características individualizables. La tasa de diagnósticos correctos se incrementó con el uso combinado de la ecografía convencional y el Doppler energía.

En nuestra experiencia la mayoría de angiomiolipomas producen una imagen típica, patognomónica con ecografía convencional, no aportando el Doppler más datos; Este puede ser de utilidad para la confirmación de pequeñas masas renales ayudando al diagnóstico diferencial con los pseudotumores estableciendo el mapa de vascularización cortical y permitiendo la detección de zona avasculares.

La historia natural de estas masas de pequeño tamaño no ha sido investigada debido a que la mayoría de los pacientes son tratados quirúrgicamente en el momento de la detección. Este tipo de lesiones tienden a ser de bajo grado y bajo estadio y la supervivencia a 5 años es superior al $85 \%$ (25). Varios trabajos han estudiado su evolución y demuestran que tiene un crecimiento lento y no desarrollan metástasis durante el periodo de observación (2631); esto puede ser debido a la alta proporción de tumores benignos que han sido hallados en algunas series (8-27\% de oncocitomas) (32-39). En un estudio reciente en pacientes tratados con nefrectomía parcial, en tumores menores de $2 \mathrm{~cm}$, el porcentaje de masas benignas ascendió a un $38,5 \%$ (40).

En nuestra experiencia el mayor porcentaje de pequeñas masas sólidas corresponde a angiomiolipomas y del resto el 95\% a CCR ; $5 \%$ a oncocitomas y otros tumores renales.

Volpe (40) realizó un seguimiento prospectivo de 29 pacientes con masas renales menores de 4 $\mathrm{cm}$ en pacientes no candidatos a tratamiento quirúr- gico por problemas médicos. El seguimiento medio fue de 27,9 meses y el crecimiento medio fue de 0,1 $\mathrm{cm} / a n ̃ o$. La tasa de crecimiento no estaba asociada con el tamaño inicial del tumor y ninguno de los pacientes desarrolló metástasis.

Bosniack (27) estudió a 37 pacientes con masas renales menores de $3 \mathrm{~cm}$, con un seguimiento de 3,5 años (entre 1,8y 8,5 años); el crecimiento medio fue de $0,36 \mathrm{~cm} /$ año (entre 0 y $1,1 \mathrm{~cm}$ ) y ninguno desarrolló metástasis. Intervinieron a 28 pacientes, la mayoría de lesiones fueron grado I y ninguna grado III. De los tres que crecieron rápidamente, 2 fueron grado II.

Kato (41) observó a 18 pacientes durante 12-63 meses con este tipo de masas que finalmente se extirparon. La media de crecimiento fue de 0,42 $\mathrm{cm} /$ año y comprobó que existía una correlación entre el grado tumoral y el crecimiento; así en los de grado III fue de 0,93 cm/año de media.

Redon (26) realizó un estudio con 13 pacientes a los que observó 42 meses con masas de pequeño tamaño. Únicamente 2 crecieron rápidamente, en el resto el crecimiento medio fue de 0,14 cm/año. Ninguno desarrolló metástasis.

El demostrado lento crecimiento y bajo grado de estos tumores permite cuando el riesgo quirúrgico es elevado, la edad avanzada o los estudios radiológicos inespecíficas realizar un estrecho seguimiento y adoptar la decisión más adecuada según su evolución jugando la ecografía un papel importante en el seguimiento del diámetro tumoral. Cuando se cumplen estrictamente los criterios ultrasonográficos de angiomiolipoma pensamos que no es obligada la realización de otras exploraciones siendo precisa su evaluación periódica para objetivar su modificación morfológica. En el resto la TAC o RNM confirman su existencia y naturaleza.

\section{BIBLIOGRAFIAA y LECTURAS RECOMENDADAS ( ${ }^{*}$ lectura de interés $y^{* *}$ lectura fundamental)}

1. MOTZER, R.J.; BANDER, N.H.; NAMUS, D.M.: "Renal-cell carcinoma". N. Engl. J. Med., 335: 865, 1996.

2. HÉLÉNON, O.; CORREAS, J.M.; BALLEYGUIER, C. y cols.: "Ultrasound of renal tumors". Eur. Radiol., 11: 1890, 2001.

3. SKINNER,D.G.;COLVIN, R.B.; VERMILLION, C.D. y cols.: "Diagnosis and management of renal cell carcinoma. A clinical and pathologic study of 309 cases". Cancer, 28: 1165, 1971. 
4. KONNAK, J.W.; GROSSMAN, H.B.: "Renal cell carcinoma as an incidental finding". J. Urol., 134: 1094, 1985.

5. BRETHEAN, D.; LECHEVALLIER, E.; EGHAZARIAN, C. y cols.: "Pronostic significance of incidental renal cell carcinoma". Eur. Urol., 27: 319, 1995.

6. PATARD, J.J.; RODRÍGUEZ, A.; RIOUX-LECREREK, N. y cols.: "Significación pronóstica del modo de detección en los tumores renales". BJU International, 90: 358, 2002.

7. HELLSTEN, S.; BERGE, T.; WEHLIN, L.: "Unrecognized renal cell carcinoma: Clinical and diagnostic aspects". Scand. J. Urol. Nephrol., 15: 269, 1981.

8. TSUI, K.H.; SHVARTS, O.; SMITH, R.B. y cols.: "Renal cell carcinoma: prognosis significance of incidentally detected tumors". J. Urol., 163: 426, 2000.

9. THOMPSON, I.M.; PEEK, M.: "Improvement in survival of patients with renal cell carcinomaThe role of the serendipitiously detected tumor". J. Urol., 140: 487, 1988.

10. PULS, R.; HOSTEN, N.; LEMKE, M. y cols.: "Perfusion abnormalities of kidney parenchyma: microvascular imaging with contrast enhaced color and power Doppler ultrasonography. Preliminary results". J. Ultrasound Med., 19: 817, 2000.

11. SIM, J.S.; SEO, C.H.; KIM, S.H. y cols.: "Differentition of small hyperechoic renal cell carcinoma from angiomiolipoma: computed-aided tissue echo quantification". J. Ultrasound Med., 18: 261, 1999.

12. MAYAYO, T.; RIVAS ESCUDERO, J.A.; GALMES BELMONTE, I. y cols.: "Posibilidades de la ecografía en los algoritmos diagnósticos urológicos". Arch. Esp. Urol., 48: 665, 1995.

13. ESCUDERO BARRILERO, A.; ROMERO, J.; MAYAYO, T. y cols.: "El valor de la punción translumbar en el diagnóstico del quiste hidatídico renal”. Rev. Clin. Esp., 162: 179, 1981.

14. GHARBI, H.A.; HASSINE, W.; BRAUNER, M.W. y cols.: "Ultrasound examination of the hydatic liver". Radiology, 139: 459, 1981.

15. GONZÁLEZ, L.R.; MARCOS, J.; ILLANAS, M. y cols.: "Radiologic aspect of hepatic echinococcosis". Radiology, 130: 21, 1979.

16. NABIZADEH, I.; MOREHOUSE, H.T.; FREED, S.Z.: "Hydatic disease of kidney". Urology, 22: 176, 1982.

17. ARAGONA, F.; DI CANDIO, G.; SERRETA, V. y cols.: "Renal hydatid disease: Report of 9 cases and discussion of urologic diagnostic procedures". Urol. Radiol., 6: 182, 1984.

18. MAYAYO, T.; ROMERO-MAROTO, J.; MAGANTO, E. y cols.: "Sonografía y punción percutánea en la hidatidosis renal". Actas Urol. Esp., 3: 23, 1979.
19. ODEV, K.; KILINC, M.; ARSLAN, A. y cols.: "Quistes hidatidicos renales y estudio de sus imagenes radiológicas". Eur. Urol.(Edición Española), 4: 39, 1997.

20. GOINEY, R.C.; GOLDENBERG, L.; COOPERBERG, P.L.: "Renal oncocytoma: sonographyc analysis of 14 cases". Am. J. Roetgenol., 143: 1001, 1984.

21. BOSNIAK, M.A.: "The current radiological approach to renal cyst". Radiology, 158: 1, 1986.

22. SCHLICHTER, A.; SCHUBERT, R.; WERNER, W. y cols.: "How acurate is diagnostic imaging in determination of size and multifocality of renal cell carcinoma as a prerequisite for nephron-sparing surgery?" Urol. Int., 64: 192, 2000.

23. JAMIS-DOW, P.L.; LENNING, S.B.: "Small $(<3 \mathrm{~cm})$ renal masses: Detection with CT versus US and pathologic correlation". Radiology, 198: 785, 1996.

24. JINZAKI, M.; OHKUMA, K.; TANIMOTO, A. y cols.: "Small solid renal lesion: usefulness of power Doppler US". Radiology, 209: 543, 1998.

25. SWEENEY, J.; THORNHILL, J.A.; GRAIGER, R. y cols.: "Incidentally detected renal cell carcinoma: pathological features, survival trends and implications for treatment". Br. J. Urol., 78: 351, 1996.

26. RENDON, R.A.; STANIETZKY, N.; PANZARELLA, T. y cols.: "The natural history of small renal masses". J. Urol., 164: 1143, 2000.

27. BOSNIAK, M.A.; BIRNBAUM, B.A.; KINSKY, G.A.: "Small renal parenchymal neoplasms: further observations on growth". Radiology, 197: $589,1995$.

28. FUJIMOTO, N.; SUGITA, A.; TERASAWA, Y. y cols.: "Observations on the growth rate of renal cell carcinoma". Int. J. Urol., 2: 71, 1995.

29. BIRNBAUM, B.A.; BOSNIAK, M.A.; MEGIBOW, A.J. y cols.: "Observations on the growth of renal neoplasms". Radiology, 176: 695, 1990.

30. ODA, T.; MIYAO, N.; TAKAHASHI, A. y cols.: "Growth rates of primary and metastatic lesions of renal cell carcinoma”. Int. J. Urol., 8: 473, 2001.

31. WEHLE, M.J.; THIEL, D.D.; PETROU, S.P. y cols.: "Conservative management of incidental contrast-enhancing renal masses as safe alternative to invasive therapy". Urology, 64: 49, 2004.

32. SILVERMAN, S.G.; LEE, B.Y.; SELTZER, S.E. y cols.: "Small $(<3 \mathrm{~cm})$ renal masses: correlation of spiral CT features and pathologic findings". AJR., 163: 597, 1994.

33. SOLAR, D.H.; KAMMERHUBER, F.; ALTZIEBLER, S.: "Multiphasic helical CT of the kidney: increased conspicuity for detection and characterization of small $(<3 \mathrm{~cm})$ renal masses". Radiology, 202: 211, 1997. 
34. CURRY, N.S.: "Imaging the small solid renal mass". Abdom. Imaging., 27: 629, 2002.

35. ZAGORIA, R.J.: "Imaging of the small renal masses: A medical sucess story”. AJR., 175: 945, 2000.

36. HERNÁNDEZ JOVER, D.; DE LA TORRE HELGUERA, P.; ALBEROLA BOU, J. y cols.: "Masa renal pequeña. Manejo diagnóstico". Arch. Esp. Urol., 54: 593, 2001.

37. LEVINE, H.; HUNTRAKOON, M.; WETZEL, L.H.: "Small renal neoplasms: clinical pathologic and imaging features". AJR., 153: 69, 1989.

38. BOSNIAK, M.A.: "The small $(<3 \mathrm{~cm})$ renal parenchymal tumor: detection, diagnosis and controversies". Radiology, 179: 307, 1991.

39. STEINBERG, A.P.; LIN, C.H.; MARTIN, S. y cols.: "Impact of tumor size on laparoscopic partial nephrectomy: anlysis of 163 patients". J. Urol., 169: 174, 2003.

40. VOLPE, A.; PANZARELLA, T.; RENDON, R.A. y cols.: "The natural history of incidentally detected small renal masses". Cancer, 100: 738, 2004.

41. KATO, M.; SUZUKI, T.; SUZUKI, Y. y cols.: "Natural history of small renal cell carcinoma: evaluation of growth rate, histological grade, cell proliferation and apoptosis". J. Urol., 172: 863, 2004. 